\title{
Current status and future aspects of kidney transplantation in Japan
}

\author{
Atsushi Aikawa
}

\begin{abstract}
In Japan, there are very few cases of organ donation, including kidneys. The number of deceased donor kidney transplants (DDKTs) is much less than that of other developed Asian and Western countries, although donation after brain death is increasing slowly. Living donor kidney transplants (LDKTs) are more popular than DDKTs in Japan. However, the number of LDKTs per million population was still less than that in Korea, the UK, and the USA. Living donor ABO-incompatible kidney transplants have been performed in Japan since 1989, and longterm outcomes proved similar to those for ABO-compatible kidney transplants. Preemptive kidney transplants comprised $30 \%$ of LDKTs. In spite of the small number of kidney transplants, patient, and graft survival rates in Japan are superior to those of any other country.

The infusion of regulatory $T$ cells may induce immunotolerance in kidney transplants although acute rejection frequently occurred when immunosuppression was withdrawn and anti-donor-specific antibodies (DSAs) production could not always be suppressed. Combined kidney and bone marrow transplantation may induce immunotolerance, although a few recipients produced DSA.

Kidney regeneration has become a reality. Nephron progenitor cells have been generated from human-induced pluripotent stem cells, readily reconstituting three-dimensional nephrons, including vascularized glomeruli with podocytes. The niche method has been used to generate kidney, urinary tract, and bladder tissue. Many Japanese scientists are researching kidney regeneration, and a kidney regenerated from a recipient's cells could be transplanted without immunosuppression in the near future.
\end{abstract}

Keywords: Kidney transplantation, Deceased donor, Living donor, Regulatory T cell, Kidney regeneration, Japan

\section{Background}

The realization of the current status of kidney transplantation in Japan compared to that of other countries is very important [1]. In particular, deceased organ donation, including a kidney in 2016 occurred much less often in Japan than in other Asian developed countries, such as Korea and Taiwan, as well as Western countries [2]. Therefore, the mean waiting time for a deceased donor kidney transplant (DDKT) in adults is $>12$ years [3]. Medical staff and donor coordinators do not always ensure a terminal patient's will for organ donation and do not always present the option of organ donation to the patient's family in an intensive care unit and emergency department [4]. These factors appeared to be the major causes of the extremely low organ donation rate

Correspondence: aaikawa@med.toho-u.ac.jp

Department of Nephrology, Toho University Faculty of Medicine, 6-11-1 Omorinishi, Otaku, Tokyo F143-8541, Japan in Japan. A research group funded by the Japanese Ministry of Health, Welfare and Labor is considering a new approach of optional presentation of organ donation as a terminal care procedure, provided by a qualified social medical worker [5].

The number of living donor kidney transplants (LDKTs) has increased steadily in Japan; however, the number of LDKTs per million population (pmp) in 2016 in Japan was 11.6, lagging behind that of South Korea (23.0), the UK (15.6), and the USA (18.0) [6].

The number of $\mathrm{ABO}$-incompatible kidney transplants was approximately $30 \%$ of all LDKTs [7], and patient and the graft survival rates in $\mathrm{ABO}$ - incompatible kidney transplants were similar to those of $\mathrm{ABO}$-compatible transplants [8-10]. The number of preemptive kidney transplants (PEKT) was $>30 \%$ of LDKTs [11]. A nephrologist, nurse, and coordinator cooperating with an early referral of patients with chronic kidney disease (CKD) to a

(c) The Author(s). 2018 Open Access This article is distributed under the terms of the Creative Commons Attribution 4.0 International License (http://creativecommons.org/licenses/by/4.0/), which permits unrestricted use, distribution, and 
kidney transplant center could increase and provide better outcomes of PEKT.

The patient and graft survival rates of DDKT and LDKT are better than those of any other countries despite the small numbers of kidney transplants $[12,13]$. In Japan, kidney transplantation, as well as peritoneal dialysis, compared to hemodialysis, was rarely presented as an option for renal replacement therapy (RRT) [14]. Kidney transplantation should be introduced to patients who qualify as recipients. Such delivered information about RRT to patients with CKD was recently approved to be covered by medical insurance.

Recently, donor-specific immunosuppression and kidney regeneration has been developed as future aspects of kidney transplantation in Japan. Regulatory $\mathrm{T}$ cell (Treg) therapy was almost successful in liver transplants, although anti-donor-specific antibodies (DSAs) were produced in two of 10 recipients [15]. Immunotolerance is more difficult to induce in kidney than in liver transplants. However, clinical trials of Treg therapy in kidney transplantation are ongoing in Japan, as well as the USA [16, 17].

Human-induced pluripotent stem cells are being used to study kidney regeneration is now being studied using iPSCs [18]. The nephron progenitors were successfully generated and completely replaced native nephron progenitor cells with donor cells from the mixture cells produced by the niche method [19].
Furthermore, metanephroi with a bladder were retrieved successfully [20]. Kidney regeneration may become real in the near future for use as a transplant graft.

\section{Current status of kidney transplantation in Japan DDKT}

\section{Deceased organ donation}

There is a global shortage of organ donations. Compared to that of other developed Asian, as well as Western countries, the number of organ donations is too small in Japan (Fig. 1) [2]. The number of deceased organ donors pmp in 2016 was 0.8 in Japan and 4.5, 6.3, 11.2, 21.4, 28.7, 31.0, and 46.7 in Taiwan, Hong Kong, South Korea, the UK, France, the USA, and Spain, respectively (Fig. 1) [2]. The concept of brain death is accepted in the younger Japanese generation. According to research conducted by the cabinet office of the government of Japan in 2017, 41.8\% of the population had a will specifying organ donation in cases of brain death and cardiac arrest [21]. Notably, $69.6 \%$ of the approximately $18-20$-year generation had a positive donation will compared to $34.2 \%$ of the 60 -year generation [21]. In spite of this, the actual number of organ donations is far less. This discrepancy might not be realized by doctors and transplant coordinators, and may be the reason why very few present the option of organ donation to the family of brain-dead patients. The

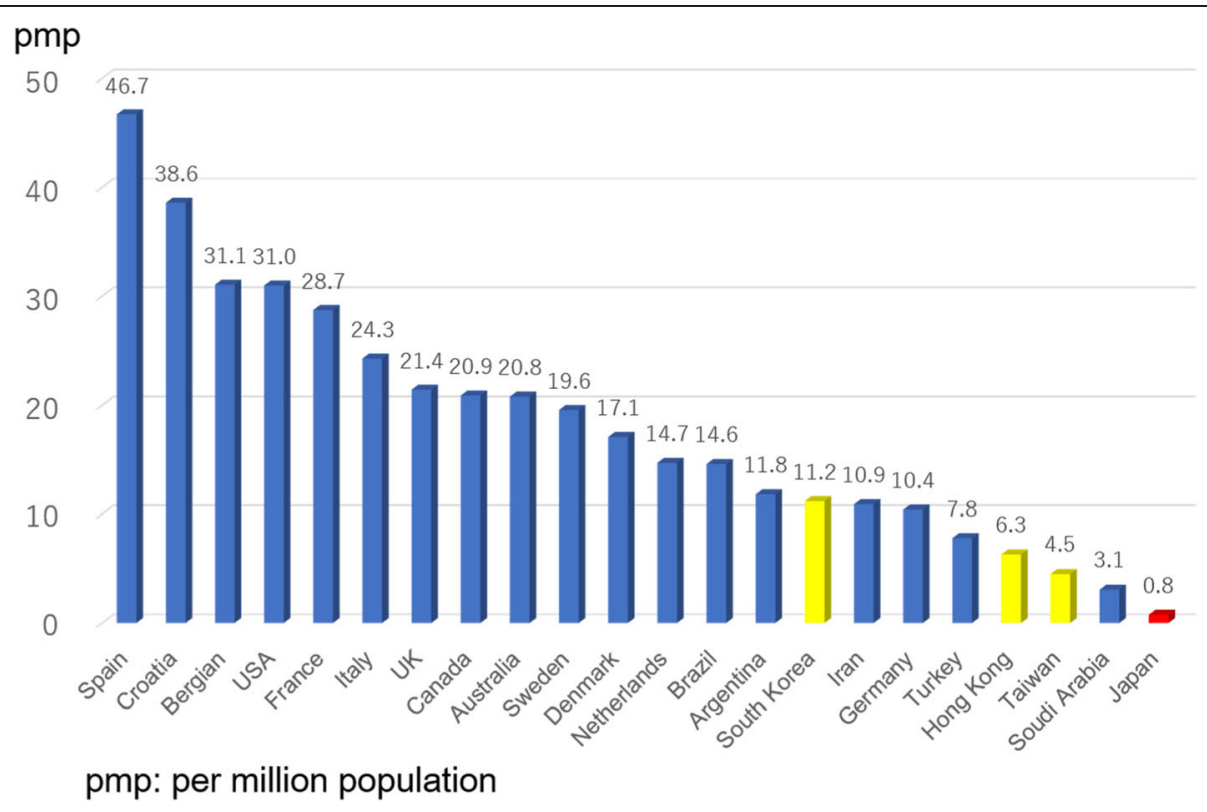

Fig. 1 Worldwide deceased organ donations pmp in 2016. pmp: per million population. This figure was referred as reference [2]. Yellow; Asian countries, red; Japan Organ donation pmp in Japan in 2016 was lower than Asian developed countries such as South Korea, Taiwan, Hong Kong, and Western countries 
United States Routine Inquiry Laws on Organ Donation (required request laws) request consent of potential candidates or their families for donation or at least inform people of the option [22]. In European countries, in-house donor coordinators ask potential donors' families to donate organs in terms of transplant procurement management [23]. However, the option for organ donation from patients with brain death is not regulated by law and depends on the decision of charged doctors in Japan [24]. In a research study of medical record reviews of 3898 cases as the Donor Action Program in donor hospitals, the rate of optional presentation of organ donation to the family was $18.9 \%$ only [4]. Consequently, organs were donated from $0.1 \%$ of brain-dead patients and $3.4 \%$ of patients with cardiac arrest [4]. To mitigate this low rate of organ donation, Yokota's research group, founded by the Japanese Ministry of Health, Welfare, and Labor, suggested that terminal and grief care includes an optional presentation of organ donation mediated by qualified medical social workers [5].

\section{The number of DDKTs and kidney donations}

The number of DDKTs in Japan has shown a gradual recent increase since 2014, but remains low, being 224 (ppm) in 2017 (Fig. 2) [25, 26]. Of these, 91 kidneys were used in kidney transplants, 35 in simultaneous pancreas-kidney transplants, and seven in simultaneous liver-kidney transplants [25]. Compared to those of other developed Asian and Western countries, the number of DDKTs in Japan remains few (Fig. 3). The number of DDKTs pmp in 2016 was 1.4 in Japan, compared to $7.9,8.2,20.7,35.3,45.1,55.9$, and 62.8 in Taiwan, Hong Kong, South Korea, the UK, the USA, France, and Spain, respectively (Fig. 3) [1]. Since the revision of the Japanese transplant law in 2010, kidney transplantation after brain death has increased slowly. In contrast, kidney transplantation after cardiac arrest has decreased remarkably since 2010 (175) to 42 in 2015 , the lowest number to date $[25,26]$. Terminal care remains unpopular in Japan. Very few medical staff actively discontinue ventilation, even in brain-dead patients. Donation after circulatory death (DCD) is either controlled or uncontrolled, as per the Maastricht classification [27]. Uncontrolled DCD refers to organ retrieval following an unexpected cardiac arrest from which the patient cannot or should not be resuscitated. In contrast, controlled DCD takes place after death following the planned withdrawal of life-sustaining treatments that have been considered of no overall benefit to a critically ill patient in the intensive care unit or emergency department. In Japan, the number of controlled DCDs is low, because although life-sustaining treatments usually continue or are minimized, respiratory support is not withdrawn until cardiac arrest. Therefore, emergency staff, retrieval doctors, and the donor family must wait for cardiac arrest in those patients still on ventilation. In most countries except Japan, organs retrieval is done at circulatory death before cardiac arrest. Donation after cardiac arrest requires intense effort and places enormous burdens on the people involved. Consequently, the quality of a retrieved kidney after cardiac arrest in Japan is not always satisfactory because of long-term hypotension

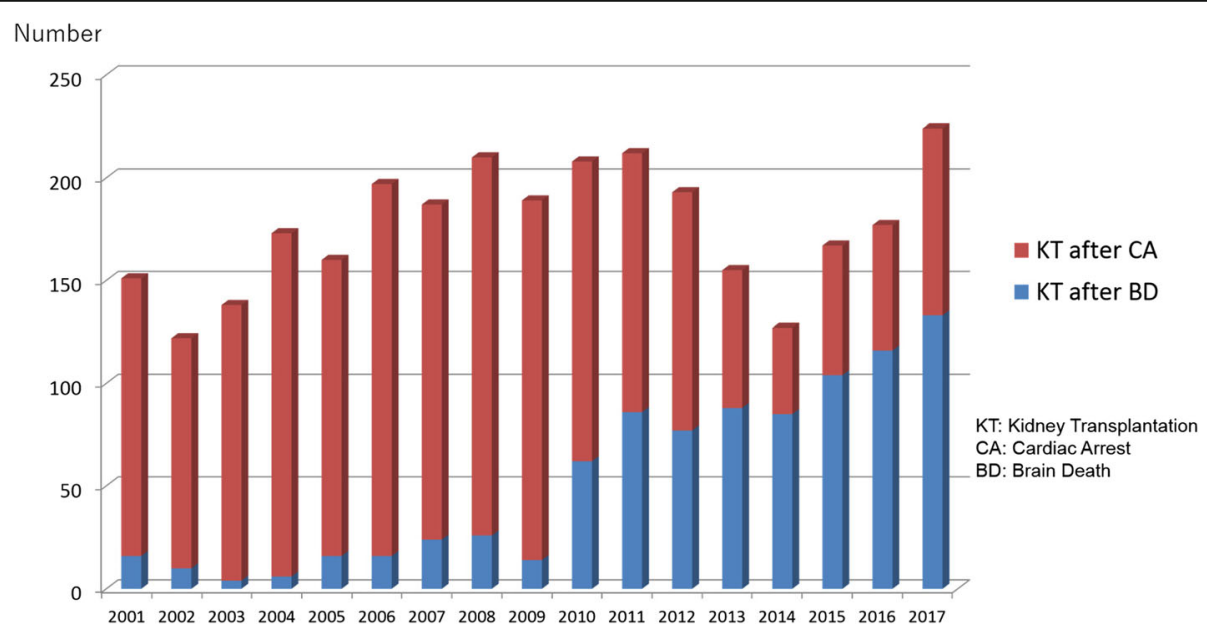

Fig. 2 Number of deceased donor kidney transplants (DDKTs) in Japan (2008-2017). This figure was referred by reference [25] and table 1 in reference [26]. The number of kidney transplants from donors after brain death has slowly increased since 2010 when the new low was enforced. In contrast, the number of kidney transplants from donors after cardiac arrest has decreased since 2010. The total number of DDKTs returned to the level of in 2017 


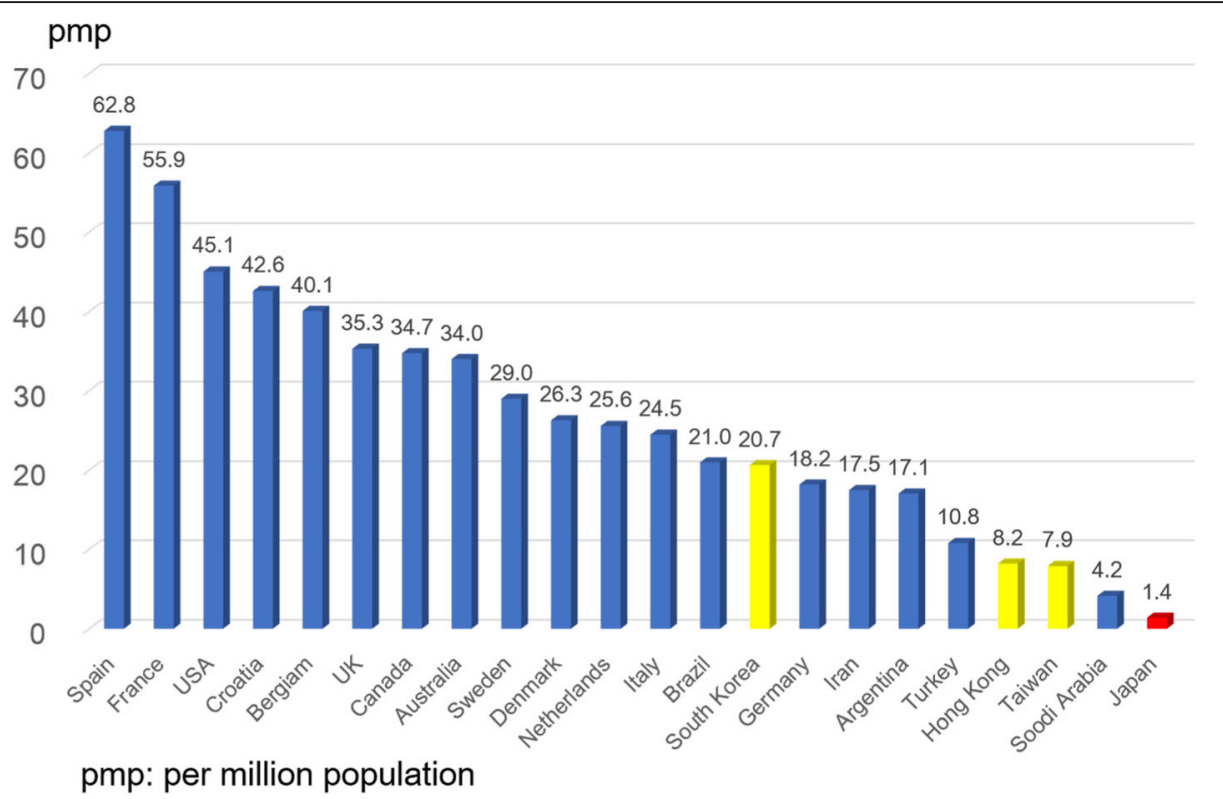

Fig. 3 Worldwide deceased donor kidney transplants pmp in 2016 pmp: per million population. This figure was referred as reference [1]. Yellow Asian countries; red Japan

and poor urination. These factors have contributed to the recent decrease in donation after cardiac arrest in Japan (0.3 ppm in 2017) $[25,26]$. In contrast, the cases of DCDs in Spain (12.3 pmp in 2017), the UK (9.2 pmp in 2016), Belgium (9.1 pmp in 2017), and the USA (5.8 ppm in 2017) have remarkably increased [28].

\section{Waiting time for DDKT}

The extreme shortage of organ donations in Japan results in prolonged waiting time for DDKTs. In 2016, the waiting times for DDKT in patients $\geq 20,16-19$, and $<16$ years old were $4486 \pm 2266,1003 \pm 605$, and $893 \pm 855$ days, respectively [3]. According to the DDKT recipient selection guideline, the waiting time is the most critical factor. Therefore, children $<16$ and 16-19 years old, respectively, score 14 and 12 points for priority, making the waiting time for children much shorter than that for adults. Age matching between donor and recipient was discussed at the 7 th Meeting of the Working Group of Kidney Transplantation [29] and the 45th Organ Transplantation Scientific Board committee meeting under the supervision of the Japanese Ministry of Health, Welfare, and Labor [3]. It was concluded that a retrieved kidney from a donor $<20$ years old should be given to a recipient $<$ 20 years old. This decision was beneficial to children waiting for DDKT.

\section{Patient and graft survival rates for DDKT}

In spite of the small numbers of DDKTs in Japan, they have a better outcome than the DDKTs of any other countries. Between 2010 and 2015, patient (791 DDKT recipients) and graft (739 DDKT recipients) survival rates were $97.9 \%$ and $96.5 \%$, respectively, at 1 year and $92.7 \%$ and $87.3 \%$, respectively, at 5 years (Fig. 4a, b) [30]. Patient and graft DDKT survival rates have improved significantly compared to those from previous periods (Fig. 4a, b). In contrast, from 2008 to 2015, patient survival rates in the USA were $96.2 \%$ at 1 year $(42,532$ DDKT recipients) and $83.1 \%$ at 5 years $(29,226$ DDKT recipients) [12], while graft survival rates were $93.2 \%$ (41,992 DDKT recipients) and $74.4 \% \quad(27,950$ DDKT recipients), respectively [30]. In spite of the differences in the number of recipients and data timespan, it appears that patient and graft DDKT survival rates in Japan are better than those in the USA.

\section{LDKT}

\section{The number of LDKTs}

The number of LDKTs in Japan has gradually increased from 1043 in 2007 to 1471 in 2016 (Fig. 5) [26]. The number of LDKTs pmp in 2016 was 11.6 in Japan, and 2.5, 4.6, and 23.0 in Hong Kong, Taiwan, and South Korea, respectively (Asian developed countries) (Fig. 6) [6]. The number of LDKTs pmp has increased even in 


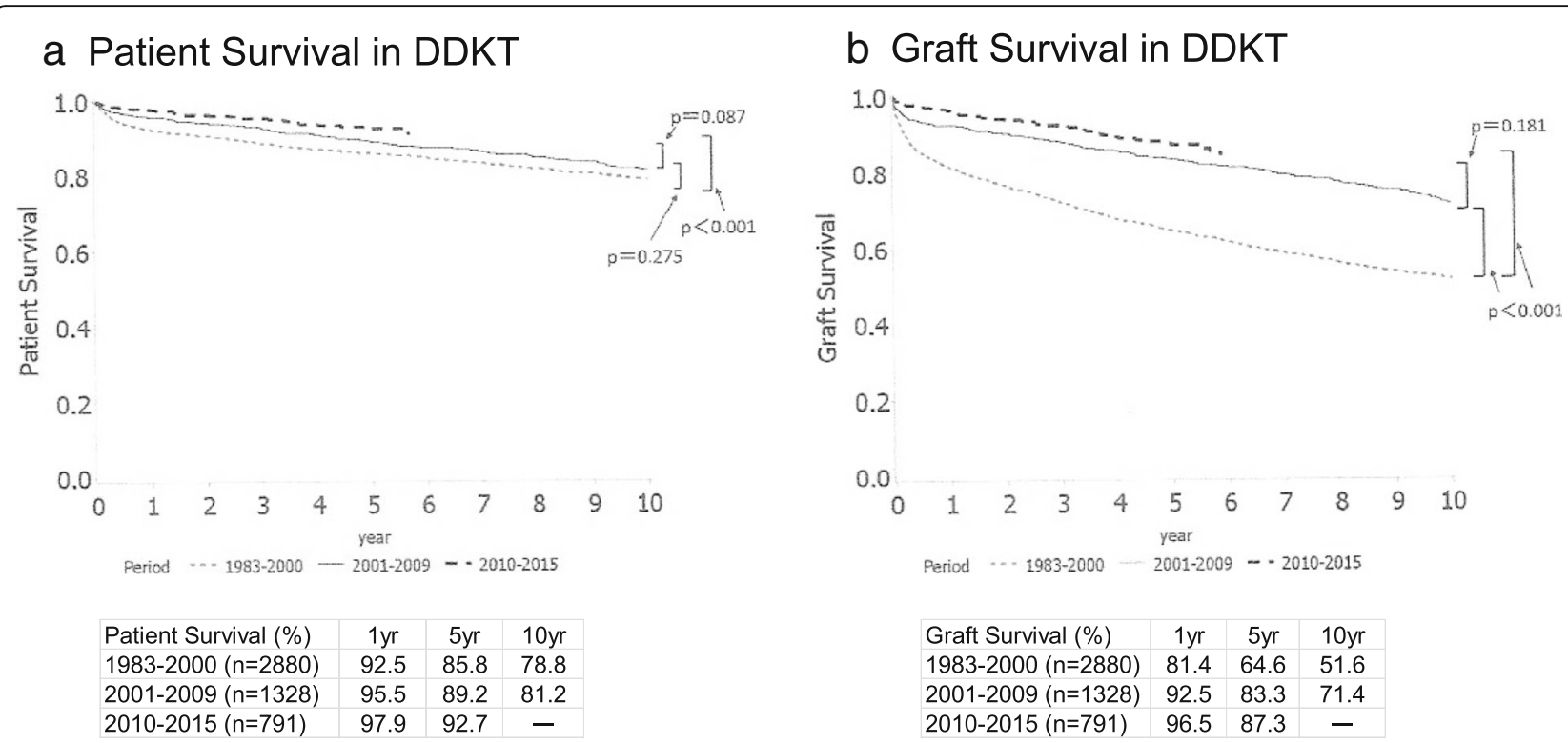

Fig. 4 Patient and graft survival rates in deceased donor kidney transplants in Japan. Figures were modified from figure 1 and 2 in reference [12] and table 20 in reference [12]

Western countries such as the Netherlands, Denmark, the USA, the UK, and Sweden $(30.4,18.6,17.6,15.6$, and 14.5, respectively) (Fig. 6) [6].

\section{Patient and graft survival rates for LDKT}

LDKT outcomes in Japan are better than those in any other country. From 2010 to 2015, the 1- and 5-year patient (6283 LDKT recipients) survival rates were 99.2\% and $97.4 \%$, respectively, and graft (5888 LDKT recipients) survival rates were $98.7 \%$ and $94.5 \%$, respectively (Fig. 7a, b) [12]. Patient and graft LDKT survival rates improved significantly compared to those from previous periods (Fig. 7a, b) [12]. In contrast, from 2008 to 2015, the USA patient survival rates were

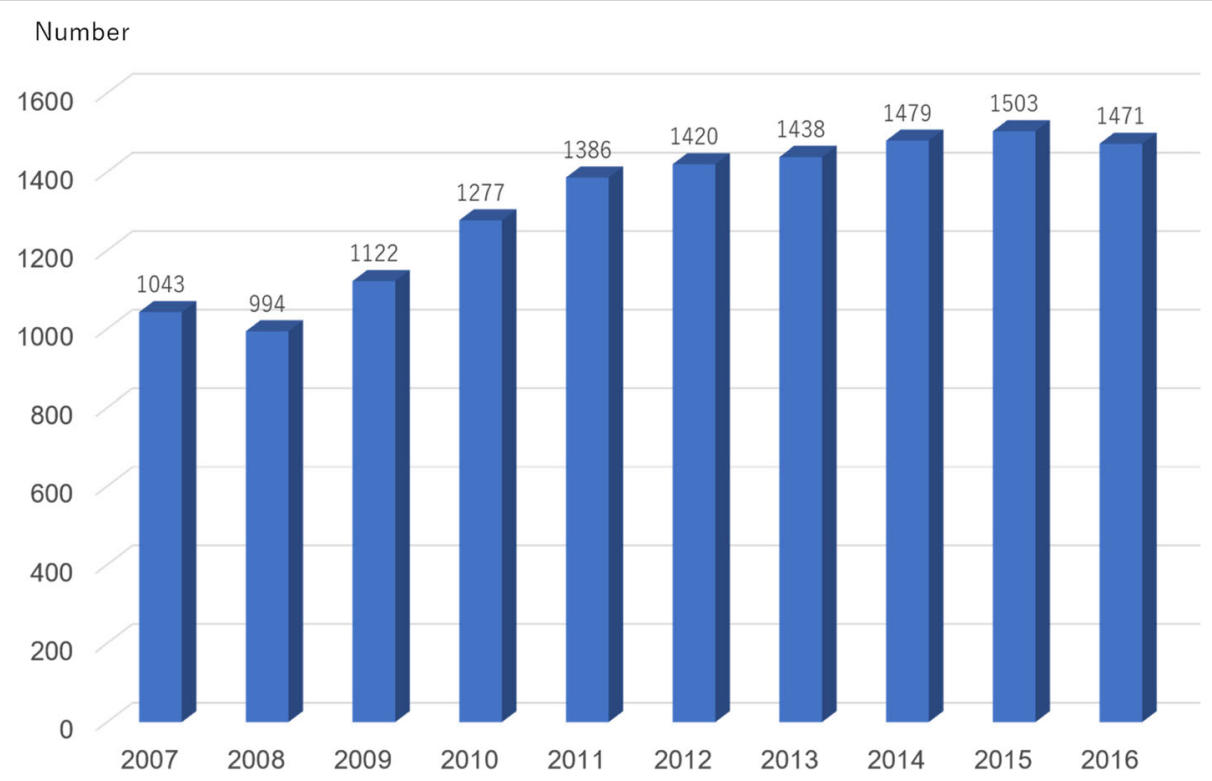

Fig. 5 Number of living donor kidney transplants in Japan (2007-2016). This figure was modified form table 1 in reference [26] 


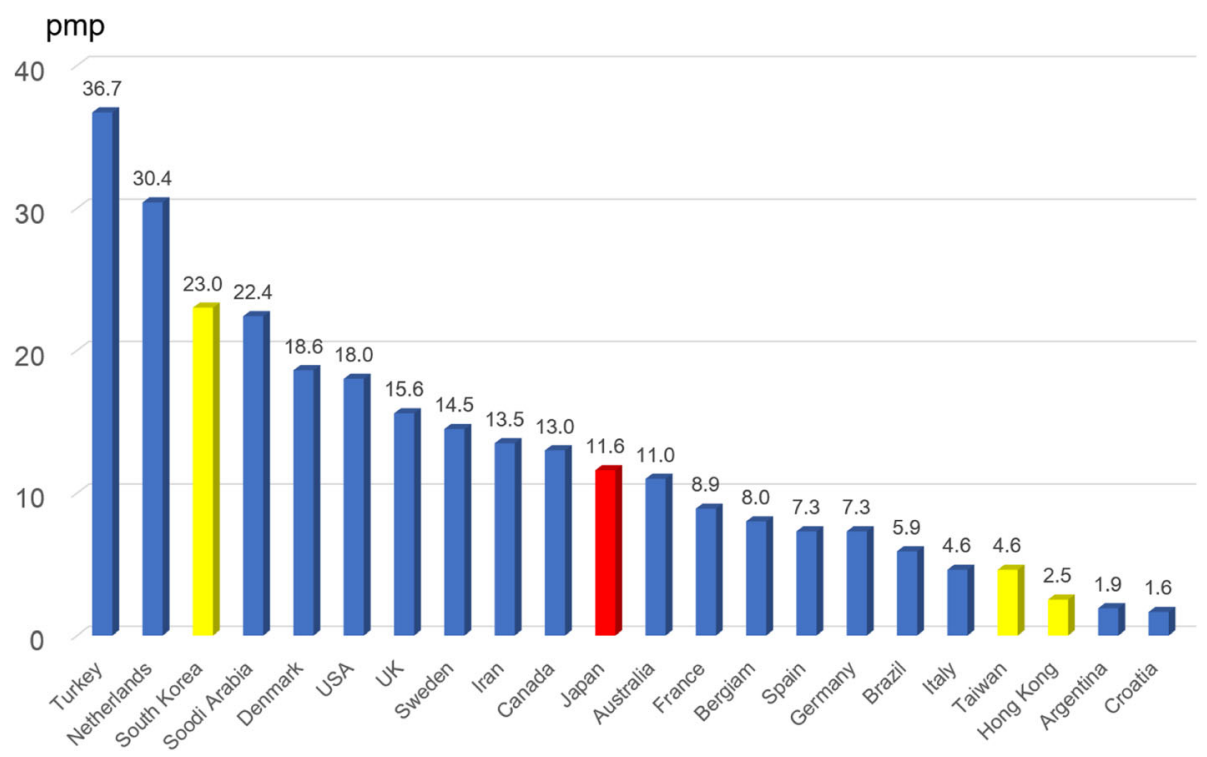

Fig. 6 Worldwide living donor kidney transplants pmp in 2016. pmp: per million population. This figure was modified from reference [6]. Yellow; Asian countries, red; Japan

$98.8 \%$ at 1 year (21,584 LDKT recipients) and $92.0 \%$ at 5 years $(18,824$ LDKT recipients), while graft survival rates were $97.5 \%$ (21,446 LDKT recipients) and 85.6\% (18,259 LDKT recipients), respectively [30]. Patient and graft survival rates of LDKTs in Japan appeared to be better than those in the USA in spite of the differences in the number of recipients and the data timespan.

\section{ABO-incompatible LDKTs}

In 2016, the number of ABO-incompatible LDKTs was 390 (29.3\%) among 1331 LDKTs [7]. At Toho University, Japan, we have performed ABO-incompatible LDKTs since 1989. Contrary to negative expectation, hyperacute rejection rarely occurred. Desensitization therapy for suppression of a donor blood group

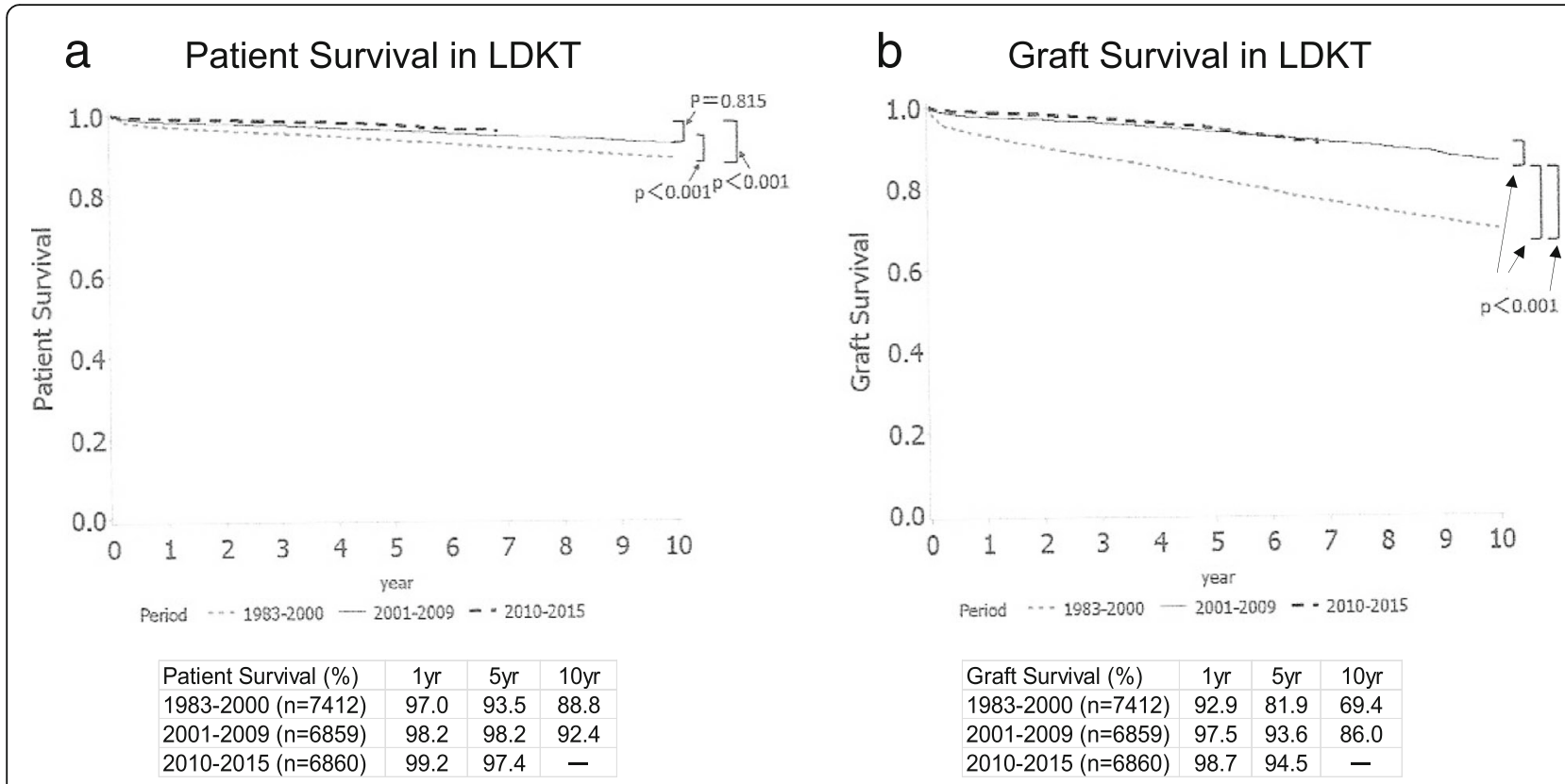

Fig. 7 Patient and graft survival rates in living donor kidney transplants in Japan. Figures were modified from figures 1 and 2 in reference [8] and table 20 in reference [12] 
antibody in ABO-incompatible LDKT consists of rituximab, mycophenolate mofetil, calcineurin inhibitor, and steroids, as well as antibody removal procedures, such as double filtration plasmapheresis (DFPP) and plasma exchange (PE) using $\mathrm{AB}$ blood group plasma. Since 2016, rituximab has been covered by medical insurance for ABO-incompatible LDKTs in Japan and is an effective antibody production suppressant. Therefore, splenectomy is no longer considered first-line therapy. Recently, DFPP and PE as preoperative conditioning were excluded in ABO-incompatible LDKT recipients with low titers of anti-donor blood group antibody IgG $(<128)[9]$ because no hyperacute rejection was apparent and good outcomes were observed unless these patients were treated with DFPP and PE [9]. This decision was of great benefit to children on peritoneal dialysis without blood access. The respective graft survival rates of ABO-incompatible $(n=86)$ and compatible $(n=300)$ LDKTs from 2006 to 2016 were $98.7 \%$ and $98.0 \%$ at 1 year, $97.3 \%$ and $96.4 \%$ at 5 years, and $97.3 \%$ and $89.2 \%$ at 10 years in a Toho University single center study (not published; Fig. 8). This study to collect and analyze the data in ABO-incompatible and compatible LDKTs was approved by the ethics committee of Toho University Omori Medical Center (no. 27-236) on February 4, 2016.
Long-term outcomes of ABO-incompatible LDKTs including pediatric recipients were similar to those of ABO-compatible LDKTs [8-10].

\section{Preemptive LDKTs (PEKTs)}

PEKTs are popular in Japan. Among the 1331 LDKT recipients in Japan in 2016, $16.5 \%$ were not on dialysis and $17.4 \%$ had received dialysis a few times before transplant [11]. Approximately one-third of LDKTs in Japan in 2016 were preemptive. A patient with CKD stage G4 must be informed of the optional presentation of RRT, including KT and referred to a kidney transplant center in preparation for a PEKT [31]. At the same time, a potential living donor also should start to be explained the procedure and studied for indication as a donor. Harada et al. [32] reported that more than half of patients were referred to transplant facilities when their eGFR fell to $<15 \mathrm{~mL} / \mathrm{min} / 1.73 \mathrm{~m}^{2}$ and only $20 \%$ could actually undergo PEKT at most facilities. They concluded that most facilities required earlier referral, in cases of CKD stages G3 or G4, which was a clear difference between referrals from CKD clinicians and transplant facilities [32]. Kidney transplantation would be a first-line treatment of end-stage renal disease, if a patient with CKD would be indicated for it. However, it is rarely presented as

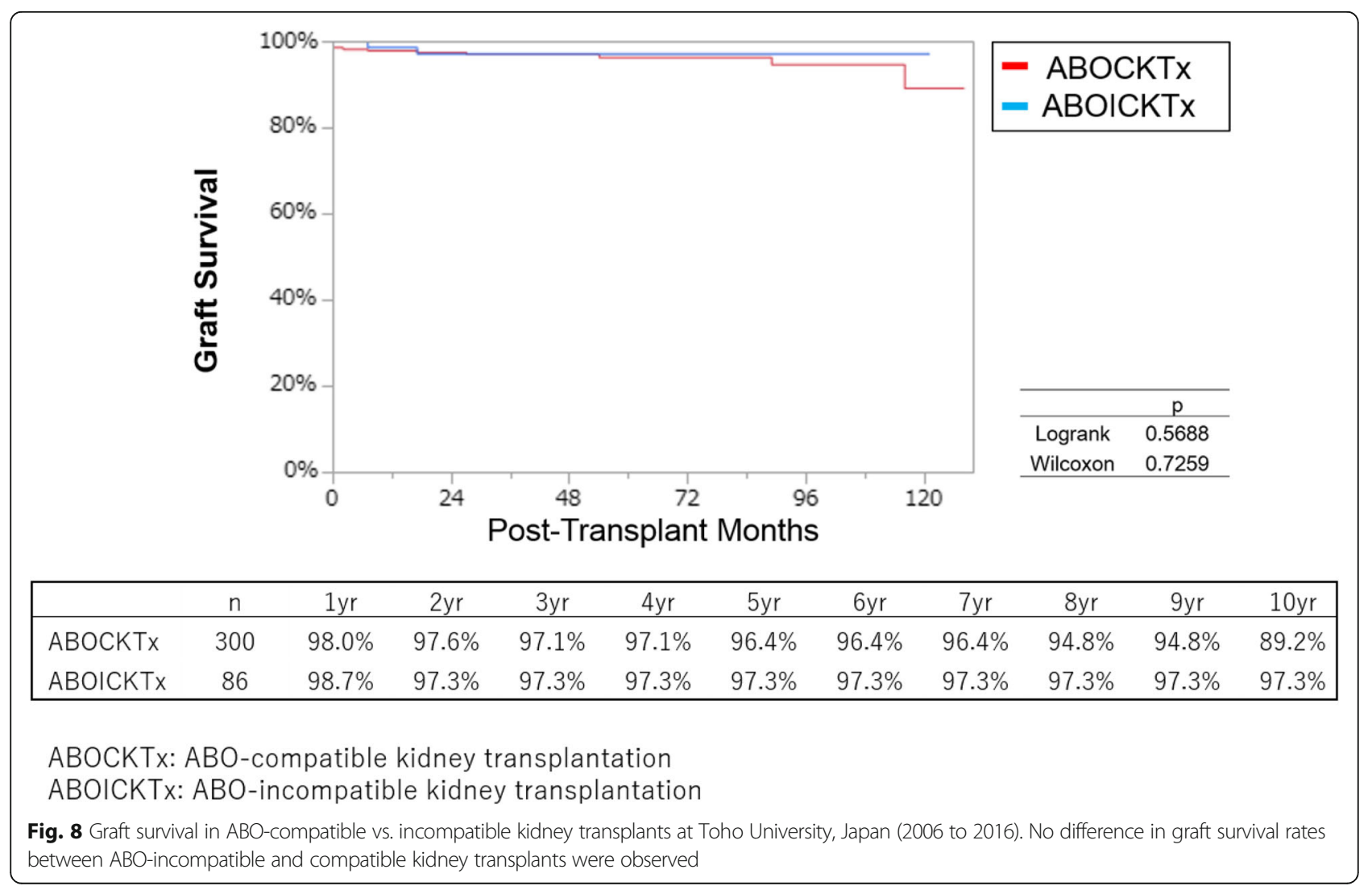


the best option for RRT [14]. Therefore, a nephrologist, nurse, and coordinator should notice better care for patients with CKD and cooperate with early referral to a kidney transplant center.

\section{Age of a recipient and a donor and their familial relationship}

The ages of LDKT recipients in $2016(n=1331)$ and 2006 $(n=939)$ were $45.7 \pm 15.4$ and $40.0 \pm 15.1$ years, respectively [33, 34]. LDKT donors in 2016 were significantly older $(P<0.0001)$ than those in $2006(57.4 \pm 11.2$ vs. 54.2 \pm 11.2 years) $[35,36]$. The proportions of LDKT recipients aged $60-69$ in 2016 and 2006 were $19.5 \%$ and $8.1 \%$, respectively [33, 34].

The familial relationships of living donors to recipients were $52.2 \%$ and $35.8 \%$ parental and $27.4 \%$ and $38.0 \%$ spousal in 2006 and 2016, respectively [37, 38]. LDKT recipients and donors tended to be older year-on-year, and a living donor tended to be a spouse more frequently than a parent. Some older patients waiting for DDKT received a LDKT from a spouse because of the prolonged DDKT waiting time.

\section{Original end-stage renal disease in kidney transplant recipients}

The original diseases of LDKT and DDKT recipients in Japan in 2016 were glomerular nephritis (26\% and $31 \%)$, diabetes mellitus (17.4\% and $12.3 \%)$, and hereditary disease (9.3\% and 7.1\%), respectively [39]. Glomerular nephritis includes IgA glomerular nephritis (IgAGN; $13.5 \%$ in 2016), focal glomerular sclerosis (FSGS; $2.2 \%$ in 2016), and others. FSGS and IgAGN frequently recur in renal grafts. Rituximab is used prophylactically to avoid FSGS recurrence in some recipients, although it is not covered by medical insurance. Tonsillectomy with steroid pulse therapy is widely accepted as treatment for a recipient with IgAGN recurrence in Japan, although tonsillectomy is not indicated in other countries. Hereditary disease includes polycystic kidney disease (6.1\%), Alport syndrome (1.1\%), and nephronophthisis $(0.9 \%)$ [39].

\section{Causes of death and graft deterioration in kidney transplant recipients after transplantation}

The primary cause of death in kidney transplant recipients from 2010 to 2015 was infection (19.8\%), followed by malignancy (14.8\%) and heart disease (11.1\%) [40]. Recently, viral infections have occurred in kidney transplant recipients more frequently than bacterial infections. Life-threatening infections include cytomegalovirus in a seronegative recipient, herpes zoster virus in a recipient without vaccination, and Epstein Barr virus-related post-transplant lymphoproliferative disease [40]. In all the periods, the primary cause of graft deterioration was chronic followed by acute rejection. Recurrence of original disease was minimal (2.4\%-4.9\%). Chronic rejection included chronic allograft nephropathy and chronic vascular rejection. Chronic allograft nephropathy was caused by hypertension, diabetes, calcineurin inhibitor toxicity, and aged kidney grafts. Chronic vascular rejection occurred mainly due to DSA-mediated rejection. Acute cellular rejection does not occur frequently under modern immunosuppression therapies and is curable with steroid pulse therapy or anti-thymocyte globulin. However, acute antibody-mediated rejection due to DSA is challenging to control.

\section{Future aspects of kidney transplantation in Japan}

Two potential kidney transplant methods require consideration. One is donor-specific immunosuppression to induce immunotolerance, thereby providing ideal conditions for organ transplantation without conventional immunosuppression. Another is the use of regenerative medicine to produce a whole kidney. Immunosuppression would not be required because this would originate from the recipient's cells.

\section{Donor-specific immunosuppression: induction of tolerance \\ Regulatory Treg therapy}

Todo et al. [15] researched the induction of immunotolerance using Treg therapy in living donor liver transplants. Adoptive transfer of an ex vivo-generated regulatory $\mathrm{T}$ cell-enriched cell product was performed in 10 consecutive adults in the early stages after liver transplant. The cells were generated using a 2-week coculture of recipient lymphocytes with irradiated donor cells in the presence of anti-CD80/86 monoclonal antibodies. Successful weaning and cessation of immunosuppressive agents were observed in $70 \%$ of patients, two of whom suffered de novo anti-class II DSA (DQ7) 1 year after drug discontinuation, remaining positive after 2 years. Because a kidney displays higher immunogenicity than a liver, the induction of immunotolerance in kidney transplants is more difficult to achieve than that in liver transplants [41]. The degree of microchimerism (donor vs. recipient cells in peripheral blood) was $1 / 10^{5}$ in kidney transplant recipients, because it was detected not by in vitro hybridization but by polymerase chain reaction. [42]. In contrast, it was $1 / 10^{3}$ in liver transplant recipients because microchimerism was able to be detected by in vitro hybridization [43]. Immunotolerance is more difficult to be induced in kidney than in liver transplants [41]. Koyama et al. [16] conducted a clinical trial of immunotolerance induction using Treg therapy in 16 LDKTs using the same procedures as those used for liver transplants. Nine 
recipients had a biopsy-proven acute rejection, and all recovered from acute rejection using steroid pulse therapy. All the recipients continued immunosuppression therapy, but at a half-dose.

Clinical trials of Treg therapy in kidney transplantation remain ongoing in the Taskp UCSF and the ONE studies [17]. These studies use autologous polyclonally or donor antigen-expanded Tregs. Recent results have been promising, and Treg therapy in kidney transplantation seems feasible and safe, although the clinical trial data remain preliminary and preclinical. There are further hurdles to overcome to determine unknown factors, such as the dose and timing of the Treg infusions, immunosuppression regimen, and long-term suppression of DSAs without maintenance immunosuppression [17].

\section{Donor bone marrow transfusion}

Chen et al. [44] reported combined HLA-mismatched kidney and bone marrow transplantation. Immunosuppression consisted of rituximab, cyclophosphamide, irradiation, anti-CD2 monoclonal antibody, steroids, and tacrolimus or cyclosporine. Bone marrow transplantation was performed simultaneously with the kidney transplant. All the 10 recipients suffered transient chimerism. Immunosuppression was successfully discontinued for 4.5-11.4 years in seven patients, and the remainder required immunosuppression reinstitution after 5-8 years due to recurrence of the original disease or chronic antibody-mediated rejection. Recently, Hotta et al. [45] reported three of four cynomolgus monkey recipients were successfully withdrawn from immunosuppression after thymoglobulin, belatacept, and donor bone marrow transfusion were given 4 months after kidney transplantation. However, even this immunosuppressive protocol could not suppress production of
DSAs. Bone marrow transfusion was proved to be an effective method to induce immunotolerance in kidney transplantation; however, it still needs to improve to suppress DSAs.

\section{Kidney regeneration \\ Nephron progenitor cells}

Several groups have developed protocols to induce nephron progenitor cells from human iPSCs [18]. When the nephron progenitors generated from human iPSCs were cultured with embryonic spinal cord or Wnt-expressing feeder cells, they readily reconstituted three-dimensional nephrons, including nephric tubules with proximal-distal segmentation and glomeruli with podocytes (Fig. 9, left) [20]. Furthermore, the glomeruli were efficiently vascularized upon transplantation into mice (Fig. 9, right).

\section{Niche method}

Nephron progenitors were injected into the fetal nephrogenic zone of different animal species, including cloned pigs, where the injected cells were continuously developed at later stages of kidney development [46, 47]. Following nephron regeneration, donor and recipient cells were mixed in the regenerated kidney. Yamanaka et al. [19] succeeded in completely replacing transplanted native nephron progenitor cells with donor cells. These nephron progenitor cells developed into mature glomeruli and renal tubules, and vascularization was observed following transplantation in vivo.

\section{Stepwise peristaltic ureter (SWPU) system}

Cloned pig metanephroi were removed from embryos and syngeneically transplanted into the omenta of recipient pigs [46]. 5 weeks after transplantation, the transplanted metanephroi were $>1 \mathrm{~cm}$ long (Fig. 10a) and exhibited ureters
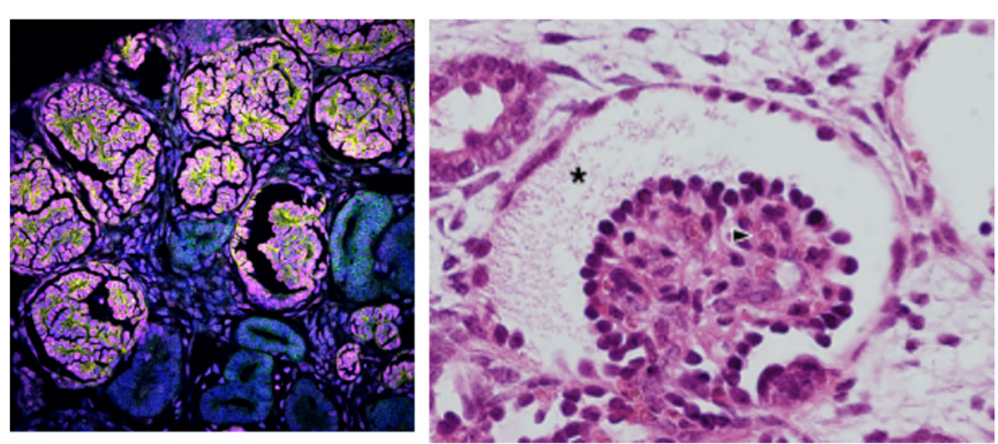

Fig. 9 Induction of nephron progenitor cells. (Left) Glomeruli and renal tubules generated in vitro from human induced pluripotent stem cells (iPSCs). The induced nephron progenitors were recombined with spinal cord feeders and cultured for 9 days. Magenta, Wt1; yellow, nephrin; green, E-cadherin. (Right) Hematoxylin-eosin staining of human iPSC-derived glomerulus vascularized upon transplantation into mice. Note the presence of red blood cells (arrowhead) in the glomerulus and eosinophilic substance in Bowman's capsule (asterisk). Photographs were referred as figures 2 and 3 in Nishinakamura and associates [20] 


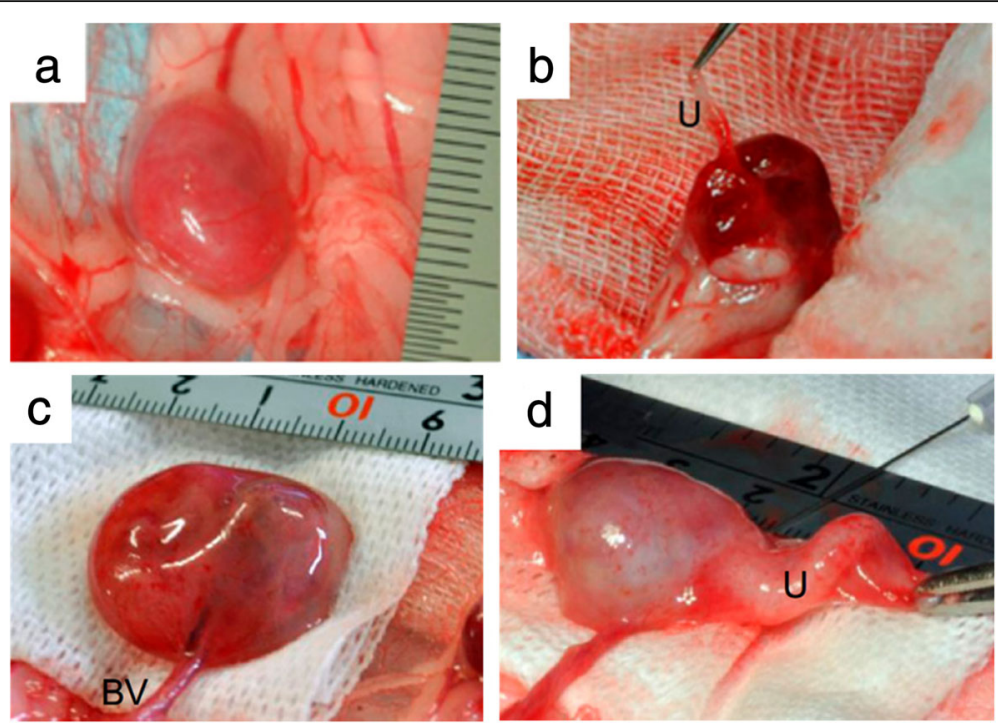

Fig. 10 Syngeneic transplantation of metanephric primordia in pigs. Cloned pig metanephroi were removed from embryos and syngeneically transplanted into the omenta of recipient pigs. 5 weeks after transplantation, the metanephroi were $>1 \mathrm{~cm}$ long (a) and exhibited ureters (b). 8 weeks after transplantation, blood vessels from the omentum were integrated into the metanephros, which was approximately $3 \mathrm{~cm}$ long (c), but the metanephros developed hydronephrosis (d). Photographs were modified from figure 1 in Yokote and associates [46]. U ureter; BV blood vessel

(Fig. 10b). 8 weeks after transplantation, blood vessels from the omentum were integrated into the metanephros, which was approximately $3 \mathrm{~cm}$ long (Fig. 10c) and produced urine, although hydronephrosis eventually was observed because of the lack of an excretion pathway (Fig. 10d). Metanephroi with bladders (cloaca) were retrieved from an E30 pig embryo and syngeneically transplanted into a pig omentum [46]. 5 weeks after transplantation, two metanephroi and a liquid-filled bladder were generated (Fig. 11, left). Anastomosis between the bladder grown from the cloacal implant and the recipient's ureter was observed (Fig. 11, right). Histopathologic analysis revealed that tubular lumina dilation and interstitial fibrosis were reduced in kidneys developed from cloacal transplants compared to metanephroi transplantation. This technique was named the SWPU system. The SWPU system may resolve two critical problems in the generation of kidneys from stem cells: construction of a urine excretion pathway and continued growth of the newly generated kidney.
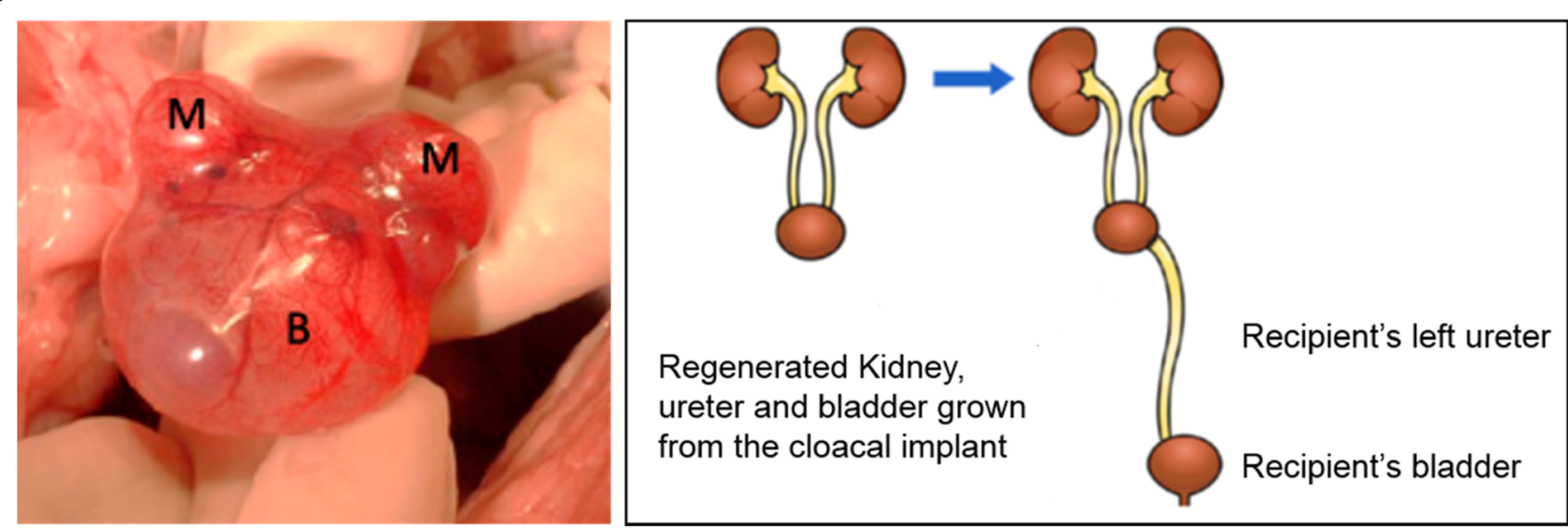

Fig. 11 Syngeneic transplantation of pig cloaca using the stepwise peristaltic ureter (SWPU) system. Pig cloaca transplanted into a pig omentum showed two metanephroi and a liquid-filled bladder 5 weeks after transplantation (left). 4 weeks after cloacal transplantation, the recipient's left ureter was connected with the bladder grown from the cloacal implant (SWPU system) (right). The photograph was modified from figure 4 in Yokote and associates [14] and the illustration modified from Yokoo and associates [43]. M metanephroi; B bladder grown from the cloacal implant 


\section{Conclusion}

The number of deceased donor organ donations, including a kidney is extremely fewer in Japan than in other developed Asian countries, as well as Western countries. A new optional presentation of organ donation as terminal and grief care by qualified medical social workers could be promising to promote DDKT.

LDKT, including ABO-incompatible and preemptive LDKT, has increased steadily; however, more development of LDKT depends on nephrologists and coworkers who inform about the opportunity for kidney transplantation.

Donor-specific immunosuppression and kidney regeneration has recently developed in Japan. Treg therapy in kidney transplants could be successful if DSA production could be suppressed. A regenerative kidney seems able to become a reality in the near future. Recipients will not require immunosuppression because a regenerative kidney is derived from the recipient's cells.

\section{Acknowledgements}

The author gratefully acknowledges the cooperation of all doctors and staff working for the Toho University Faculty of Medicine to collect and analyze the data of ABO-incompatible and compatible kidney transplants. This study was registered at the Ethics Committee of Toho University Omori Medical Center (no. 27-236) on February 4, 2016.

\section{Availability of data and materials}

The datasets used and/or analyzed are available from the corresponding author on reasonable request.

\section{Authors' contributions}

I collected and analyzed the data and wrote this review article. The author read and approved the final manuscript.

\section{Ethics approval and consent to participate}

The study of patient and graft survival rates of ABO-incompatible and compatible kidney transplants was approved by the Ethics Committee of Toho University Omori Medical Center (No. 27-236) on February 4, 2016, according to the provisions of the Declaration of Helsinki (as revised in Tokyo 2004). Informed consents were obtained from all ABO-incompatible and compatible kidney transplants recipients.

\section{Consent for publication}

Not applicable

\section{Competing interests}

The author declares that there are no competing interests.

\section{Publisher's Note}

Springer Nature remains neutral with regard to jurisdictional claims in published maps and institutional affiliations.

Received: 18 June 2018 Accepted: 2 October 2018 Published online: 28 November 2018

\section{References}

1. International registry in organ donation and transplantation. News letter 2016, Final numbers 2016: Worldwide kidney transplant from deceased donors 2016 (pmp). Transplant activity. 2017. http://www.irodat.org/img/ database/pdf/IRODaT\%20newletter\%20Final\%202016.pdf

2. International registry in organ donation and transplantation. News letter 2016, Final numbers 2016: Worldwide actual deceased organ donors 2016 (pmp). 2017. http://www.irodat.org/img/database/pdf/ IRODaT\%20newletter\%20Final\%202016.pdf.
3. Japan organ transplant network. The mean waiting days of deceased donor kidney transplants from April 1995 to December 2013, Current status of deceased donor kidney transplants Reference no. 1. p. 5. http://www.mhlw.go.jp/file/05-Shingikai-10901000-KenkoukyokuSoumuka/0000085206.pdf (in Japanese)

4. Seto K, Hasegawa T, Shinozaki N, Takahara S, Oshima S. Current status of organ donation in terms of analysis of medical record review as donor action program in donor hospitals. P138 abstract, $46^{\text {th }}$ annual meeting of Japan Society for Transplantation. Jap J Transplant. 2010;45:347 (in Japanese).

5. Ministry of health, labor and welfare. 48th Organ transplant scientific board meeting. Optional presentation method for organ donation; 2017. p. 5. reference no. 1 http://www.mhlw.go.jp/file/05-Shingikai-10601000Daijinkanboukouseikagakuka-Kouseikagakuka/0000183766.pdf (in Japanese)

6. International registry in organ donation and transplantation. News letter 2016, Final numbers 2016: Worldwide kidney transplant from living donors 2016 (pmp). Transplant activity. 2017. http://www.irodat.org/img/database/ pdf/IRODaT\%20newletter\%20Final\%202016.pdf.

7. The Japan Society for Transplantation and Japanese Society for Clinical Renal Transplantation. Table 7, Blood type and HLA compatibility, Annual progress report from the Japanese renal transplant registry: number of renal transplantations in 2016 and follow-up survey. Jap J Transplant. 2017;52:119 (in Japanese).

8. Shishido S, Hyodo YY, Aoki Y, Takasu J, Kawamura T, Sakai KK, et al. Outcomes of pediatric ABO-incompatible kidney transplantations are equivalent to ABO-compatible controls. Transplant Proc. 2012:44:214-6.

9. Aikawa A, Saito K, Takahashi K. Trends in ABO-incompatible kidney transplantation. Exp Clin Transplant. 2015;13(Suppl 1):18-22.

10. Takahashi K, Saito K, Takahara S, Okuyama A, Tanabe K, Toma H, et al. Excellent long-term outcome of ABO-incompatible living donor kidney transplantation in Japan. Am J Transplant. 2004:4:1089-96.

11. The Japan Society for Transplantation and Japanese Society for Clinical Renal Transplantation. Table 10, dialysis therapy pre-transplant, Annual progress report from the Japanese renal transplant registry: number of renal transplantations in 2016 and follow-up survey. Jap J Transplant. 2017:52:123 (in Japanese)

12. The Japan Society for Transplantation and Japanese Society for Clinical Renal Transplantation. Table 20 and Figure 1 and 2, The patient and graft survival rates in kidney transplants, annual progress report from the Japanese renal transplant registry: number of renal transplantations in 2016 and follow-up survey. Jap J Transplant. 2017; 52:129-31 (in Japanese).

13. Organ Procurement and Transplantation Network. Kidney Kaplan-Meier Graft Survival Rates for Transplants Performed: 2008-2015, National data, 2018. https://optn.transplant.hrsa.gov/data/view-data-reports/national-data/\#

14. Nakano H, Kora S, Nakamoto H, Nakayama M, Hiramatsu M, Masakane I. Society for senile patients on peritoneal dialysis (SPPD): survey on the current status of delivered informed consent for renal replacement therapy among patients with end-stage renal disease. Jpn J Nephrol. 2006;48:65863 (in Japanese)

15. Todo S, Yamashita K, Goto R, Zaitsu M, Nagatsu A, Oura T, et al. A pilot study of operational tolerance with a regulatory T-cell-based cell therapy in living donor liver transplantation. Hepatology. 2016;64:632-43.

16. Koyama I, Bashuda H, Seino K, Murakami T, Nakajima I, Fuchinoue S. Clinical trial of induction of immunotolerance in kidney transplant. Jap J Transplant. 2013:48(Suppl):201 (in Japanese).

17. Tang Q, Vincenti F. Transplant trials with Tregs: perils and promises. J Clin Invest. 2017;127:2505-12

18. Nishinakamura R, Sharmin S, Taguchi A. Induction of nephron progenitors and glomeruli from human pluripotent stem cells. Pediatr Nephrol. 2017;32:195-200

19. Yamanaka S, Tajiri S, Fujimoto T, Matsumoto K, Fukunaga S, Kim BS, et al. Generation of interspecies limited chimeric nephrons using a conditional nephron progenitor cell replacement system. Nat Commun. 2017:8:17-9.

20. Taguchi A, Kaku Y, Ohmori T, Sharmin S, Ogawa M, Sasaki H, et al. Redefining the in vivo origin of metanephric nephron progenitors enables generation of complex kidney structures from pluripotent stem cells. Cell Stem Cell. 2014;14:53-67.

21. Cabinet office, the government of Japan. Survey for public opinions concerning will of organ donation from a donor with brain death. 2018. https://survey.gov-online.go.jp/h29/h29-ishoku/index.html.

22. Andersen $\mathrm{KS}$, Fox DM. The impact of routine inquiry laws on organ donation. Health Aff (Millwood). 1988;7:65-78. 
23. Paez G, Valero R, Manyalich M. Training of health care students and professionals: a pivotal element in the process of optimal organ donation awareness and professionalization. Transplant Proc. 2009;41:2025-9.

24. Aikawa A. Comparison of organ transplantation between Japan and overseas countries--regarding new law of organ donation and transplantation. Nihon Rinsho. 2010;68:2186-94 (in Japanese).

25. Japan Organ Transplant Network. Number of transplants. 2018. https:// www.jotnw.or.jp/datafile/offer/2017.html. (in Japanese).

26. The Japan Society for Transplantation and Japanese Society for Clinical Renal Transplantation. Table 1, Kidney transplants in the last 10 years, Annual progress report from the Japanese renal transplant registry: number of renal transplantations in 2016 and follow-up survey. Jap J Transplant. 2017:52:113 (in Japanese).

27. Thuong M, Ruiz A, Evrard P, Kuiper M, Boffa C, Akhtar MZ, et al. New classification of donation after circulatory death donors definitions and terminology. Transpl Int. 2016;29:749-59.

28. International registry in organ donation and transplantation. Newsletter 2016, Final numbers 2016: Worldwide actual donors after circulatory death 2016, 2017. http://www.irodat.org/img/database/pdf/ IRODaT\%20newletter\%20Final\%202016.pdf

29. The working group of kidney transplantation under the ministry of health, welfare and labor, the government of Japan. Age matching in deceased kidney transplantation. Reference 2, The guideline to select a deceased kidney transplant recipient. The 7th meeting of the working group of kidney transplantation, 2015. http://www.mhlw.go.jp/file/05-Shingikai10901000-Kenkoukyoku-Soumuka/0000085215.pdf

30. Organ Procurement and Transplantation Network. Kidney Kaplan-Meier Patient Survival Rates and Graft Survival Rates for Transplants Performed: 2008-2015, National data, 2018. https://optn.transplant.hrsa.gov/data/viewdata-reports/national-data/

31. Yamagata K. Clinical guideline 2017 for CKD stage G3b 5. Jap J of Nephrol. 2018:59:1093-216 (in Japanese)

32. Harada H, Hotta K, Takada N, Seki T, Togashi M. Survey of practical management for induction of preemptive kidney transplantation by clinicians treating chronic kidney disease and transplant centers. J Jpn Soc Dial Ther. 2012:45:459-66 (in Japanese).

33. The Japan Society for Transplantation and Japanese Society for Clinical Renal Transplantation. Table 15, Background of donors, Annual progress report from the Japanese renal transplant registry: number of renal transplantations in 2016 and follow-up survey. Jap J Transplant. 2017;52:127 (in Japanese).

34. The Japan Society for Transplantation and Japanese Society for Clinical Renal Transplantation. Table 3, Backgrounds of recipients, Annual progress report from the Japanese renal transplant registry: number of renal transplantations in 2006 and follow-up survey. Jap J Transplant. 2007;42:416 (in Japanese).

35. The Japan Society for Transplantation and Japanese Society for Clinical Renal Transplantation. Table 15, Backgrounds of donors, Annual progress report from the Japanese renal transplant registry: number of renal transplantations in 2016 and follow-up survey. Jap J Transplant. 2017:52:127 (in Japanese).

36. The Japan Society for Transplantation and Japanese Society for Clinical Renal Transplantation. Table 4, Background of donors, Annual progress report from the Japanese renal transplant registry: number of renal transplantations in 2006 and follow-up survey. Jap J Transplant. 2007;42:416 (in Japanese)

37. The Japan Society for Transplantation and Japanese Society for Clinical Renal Transplantation. Table 5, The relation between a donor and a recipient, Annual progress report from the Japanese renal transplant registry: number of renal transplantations in 2006 and follow-up survey. Jap J Transplant. 2007:42:416 (in Japanese).

38. The Japan Society for Transplantation and Japanese Society for Clinical Renal Transplantation. Table 16, The relation between a donor and a recipient, preoperative information of living donors, annual progress report from the Japanese renal transplant registry: number of renal transplantations in 2016 and follow-up survey. Jap J Transplant. 2017:52:127 (in Japanese).

39. The Japan Society for Transplantation and Japanese Society for Clinical Renal Transplantation. Table 9, Original disease of recipients, Annual progress report from the Japanese renal transplant registry: number of renal transplantations in 2016 and follow-up survey. Jap J Transplant. 2017;52:122 (in Japanese).
40. The Japan Society for Transplantation and Japanese Society for Clinical Renal Transplantation. Table 21, Causes of death of recipients and graft deterioration, Annual progress report from the Japanese renal transplant registry: number of renal transplantations in 2016 and follow-up survey. Jap J Transplant. 2017:52:130 (in Japanese).

41. Starzl TE, Trucco M, Zeevi A, Kocova M, Ilstad S, Demetris AJ, et al. Mixed chimerism after transplantation: mechanism or marker of specific tolerance. Hepatol. 1993:17:943-5.

42. Aikawa A, Yamashita M, Arai K, Hirayama N, Ohara T, Hasegawa A, et al. Microchimerism in female renal transplant recipients from male donors. Int J Urol. 1996 Jan;3(1 Suppl):S114-9.

43. Starzl TE, Trucco M, Zeevi A, Kocova M, Ildstat S, Demetris AJ, et al. Systemic chimerism in human female recipients of male livers. Lancet. 1992:340:876-7.

44. Chen YB, Kawai T, Spitzer TR. Combined bone marrow and kidney transplantation for the induction of specific tolerance. Am J Transplant. 2014;14:1599-611.

45. Hotta K, Oura T, Dehnadi A, Boskovic S, Matsunami M, Rosales I, et al. Longterm nonhuman primate renal allograft survival without ongoing immunosuppression in recipients of delayed donor bone marrow transplantation. Transplantation. 2018;102:e128-36.

46. Yokote S, Matsunari H, Iwai S, Yamanaka S, Uchikura A, Fujimoto E, et al. Urine excretion strategy for stem cell-generated embryonic kidneys. Proc Natl Acad Sci U S A. 2015;112:12980-5.

47. Yokoo T, Fukui A, Ohashi T, Miyazaki Y, Utsunomiya Y, Kawamura T, et al Xenobiotic kidney organogenesis from human mesenchymal stem cells using a growing rodent embryo. J Am Soc Nephrol. 2006;17:1026-34.

\section{Ready to submit your research? Choose BMC and benefit from:}

- fast, convenient online submission

- thorough peer review by experienced researchers in your field

- rapid publication on acceptance

- support for research data, including large and complex data types

- gold Open Access which fosters wider collaboration and increased citations

- maximum visibility for your research: over $100 \mathrm{M}$ website views per year

At $\mathrm{BMC}$, research is always in progress.

Learn more biomedcentral.com/submissions 